\title{
Primate Insular/Opercular Taste Cortex: Neuronal Representations of the Viscosity, Fat Texture, Grittiness, Temperature, and Taste of Foods
}

\author{
Justus V. Verhagen, Mikiko Kadohisa, and Edmund T. Rolls \\ Department of Experimental Psychology, University of Oxford, Oxford OX1 3UD, United Kingdom
}

Submitted 30 March 2004; accepted in final form 27 April 2004

Verhagen, Justus V., Mikiko Kadohisa, and Edmund T. Rolls. Primate insular/opercular taste cortex: neuronal representations of the viscosity, fat texture, grittiness, temperature, and taste of foods. $J$ Neurophysiol 92: 1685-1699, 2004; 10.1152/jn.00321.2004. It is shown that the primate primary taste cortex represents not only taste but also information about many nontaste properties of oral stimuli. Of 1,122 macaque anterior insular/frontal opercular neurons recorded, $62(5.5 \%)$ responded to oral stimuli. Of the orally responsive neurons, some $(53 \%)$ represented the viscosity, tested using carboxymethylcellulose in the range $1-10,000 \mathrm{cP}$. Other neurons $(8 \%)$ responded to fat in the mouth by encoding its texture (as shown similar responses to nonfat oils), and $8 \%$ responded to gritty texture. Some neurons $(35 \%)$ responded to the temperature of the liquid in the mouth. Some neurons responded to capsaicin, and others to fatty acids. Some neurons $(56 \%)$ had taste responses. Some $(50 \%)$ of these neurons were unimodal, responding to one of these types of stimulus, and the majority combined responsiveness to these types of stimulus, with $23 \%$ responding for example to both taste and temperature. Some neurons respond to taste, texture, and temperature unimodally, but others combine these inputs. None of these orally responsive neurons responded to odor or to the sight of food. These results provide fundamental evidence about the information channels used to represent the taste, texture, and temperature of food in the first cortical area involved in taste in the primate brain. The results are relevant to understanding the physiological and pathophysiological processes related to how the properties of oral stimuli are represented in the brain and thus to the control of food intake and food selection.

\section{IN T R O D U C T I O N}

The macaque primary taste cortex is in the anterior insula and adjoining frontal operculum (abbreviated to AI/FO taste cortex) as shown by the anatomical inputs to these regions from the thalamic taste nucleus, VPMpc (the parvicellular division of the ventroposteromedial thalamic nucleus) (Pritchard et al. 1986). A remarkable difference from the taste system of rodents is that in primates there is a direct projection from the first central relay, the nucleus of the solitary tract (NTS), to the gustatory thalamus (Beckstead et al. 1980; Norgren 1984; Pritchard et al. 1989). In rodents, there is an obligatory relay from the NTS to the pontine parabrachial taste nuclei $(\mathrm{PBN})$, which in turn project to the thalamus (Norgren 1984; Norgren and Leonard 1973). The pontine taste nuclei also project to the hypothalamus and amygdala in rodents (Norgren 1976), providing direct access in rodents to these subcortical structures important in motivational behavior (e.g., feeding) and learning (Rolls 1999). In contrast, in primates there appears to be no such direct pathway from the brain stem

Address for reprint requests and other correspondence: E. T. Rolls, Dept. of Experimental Psychology, University of Oxford, S. Parks Rd., Oxford OX1 3UD, UK (E-mail: Edmund.Rolls@psy.ox.ac.uk); (Web page: www.cns.ox.ac.uk). taste areas to the hypothalamus and amygdala (Norgren 1984), and instead taste information reaches structures such as the amygdala and orbitofrontal cortex from the primary taste cortex (Baylis et al. 1994; Turner et al. 1980). This fundamental difference in the anatomy of the rodent and primate taste pathways shows that even in a phylogenetically old system such as taste, the way in which the system functions and processes information may be different across mammalian orders. Because of its potentially greater relevance to understanding the taste system in humans, we therefore analyzed the responses of neurons in the macaque primary taste cortex in which the gustatory responses of single neurons have been analyzed previously (Rolls et al. 1988; Scott and PlataSalaman 1999; Scott et al. 1986a, 1991; Yaxley et al. 1988, 1990)

The particular aims of the investigation described here were to examine whether the primary taste cortex also receives and represents other information about the properties of oral stimuli, including their viscosity, fat texture, and temperature; and if so, whether this information is represented independently of taste information (that is, by separate neurons) and whether some neurons combine information about taste and these other oral properties, as such neurons would potentially provide a neural basis for behavioral responses that could be selective of particular combinations of taste and these other oral properties. Another aim was to determine whether fatty acids are represented in the primary taste cortex, and if so, if the representation is separate from that of fat texture and of acid. A further aim was to determine whether gritty oral texture is represented separately from these other properties of oral stimuli. Part of the interest of these investigations is that all these properties contribute to the oral palatability of food and that understanding the factors that determine the palatability of food is currently of great importance given the role of palatability in the control of food intake and the rapidly increasing incidence of obesity, which is accompanied by serious health risks (Berthoud 2003; Steinberger and Daniels 2003). Another part of the interest of the investigations is that given that some neurons in the orbitofrontal cortex and amygdala do show convergence from some of the different sensory properties of oral stimuli (such as taste, texture, and temperature), it is of interest to investigate whether this convergence happens for the first time in these secondary taste areas in primates or whether the convergence is present in some neurons in the primary taste cortex. Finally, an aim was to determine whether olfactory and orally related visual stimuli (such as the sight of food) are

\footnotetext{
The costs of publication of this article were defrayed in part by the payment of page charges. The article must therefore be hereby marked "advertisement" in accordance with 18 U.S.C. Section 1734 solely to indicate this fact.
} 
represented in the primary taste cortex or whether this type of convergence is left to the secondary taste cortex in the orbitofrontal cortex (Rolls et al. 2003b; Kadohisa et al 2004a,b), where we know that single neurons reflect these types of convergence (Critchley and Rolls 1996; Rolls and Baylis 1994; Rolls et al. 1996; Thorpe et al. 1983).

\section{METHODS}

\section{Subjects}

The recordings were made in two rhesus macaques (Macaca mulatta) (1 female weighing $4.3 \mathrm{~kg}$ and 1 male weighing $6.1-6.7 \mathrm{~kg}$ ). The monkeys were pair-housed in foraging home cages. To ensure that the macaques were willing to ingest the test foods and fluids during the recording sessions, they were on mild food (150 $\mathrm{g}$ of nutritionally balanced mash plus fruits, boiled chicken eggs, nuts, seeds, and popcorn) and fluid ( $1 \mathrm{~h} /$ day ad libitum water) deprivation in that both were provided after the daily recording session. The monkeys showed steady increases in bodyweight. All procedures, including preparative and subsequent ones, were carried out in accordance with the National Institutes of Health Guide for the Care and Use of Laboratory Animals, and were licensed under the UK Animals (Scientific Procedures) Act, 1986.

\section{Recordings}

Recordings were made from single neurons in the insula and frontal operculum, in the region defined as primary taste cortex (Pritchard et al. 1986) and in which we have recorded taste neurons previously (Rolls et al. 1988; Scott et al. 1986a; Yaxley et al. 1988, 1990). The recordings were made with epoxylite-coated single neuron tungsten microelectrodes (Frederic Haer, St. Bowdoinham, ME; unzapped, 5-10 $\mathrm{M} \Omega$ at $1 \mathrm{kHz}$ ). After several tracks when the impedance had fallen $<2 \mathrm{M} \Omega$, we recoated the electrodes with epoxylite $(6001 \mathrm{M}$, Epoxylite, Bradford, UK) resulting in 5-10 M $\Omega$ impedance and good isolation (Verhagen et al. 2003a). The signal-to-noise ratio was typically $\geq 3: 1$.

For on-line monitoring of neural activity and for determining the randomized permutation stimulus sequence during an experiment, a computer (Pentium) with real-time digital and analogue data acquisition collected spike arrival times and displayed a peristimulus time histogram and rastergrams and displayed the number of spikes in 1and 3 -s poststimulus periods. To ensure that the recordings were made from single cells, the interspike interval was continuously monitored to make sure that intervals of $<2 \mathrm{~ms}$ were not seen, and the waveform of the recorded action potentials was continuously monitored. The data were also collected using a Datawave Discovery (Tucson, AZ) system, which digitized the signal (12 bit, $16 \mathrm{kHz}$ ) for $8 \mathrm{~s}$ after stimulus onset. The spikes were sorted off-line using the cluster cutting method provided with the Datawave system, and this procedure was straightforward as the data were collected with single neuron microelectrodes that typically recorded from only one neuron at a time with a high signal-to-noise ratio $(>3: 1)$. The recording sessions lasted $4-6 \mathrm{~h}$ and were conducted daily. To prevent visual associative input from evoking neural activity, we prevented the monkeys from seeing the stimuli and experimenter by a view-obstructing screen.

\section{Localization of recordings}

$\mathrm{X}$-radiography was used to determine the position of the microelectrode after each recording track relative to permanent reference electrodes and to the anterior sphenoidal process. This is a bony landmark the position of which is relatively invariant with respect to deep brain structures (Aggleton and Passingham 1981). On each track, one X-ray in the coronal plane, and one in the saggital plane, was taken. Microlesions made through the tip of the recording electrode during the final tracks were used to mark the location of typical units. These microlesions, together with the associated X-radiographs, allowed the position of all cells to be reconstructed in the $50-\mu \mathrm{m}$ brain sections with the methods described by Feigenbaum and Rolls (1991).

\section{Stimuli}

The neurons of the taste cortex were tested for their responsiveness to the set of taste, viscosity, gritty, oily stimuli, and capsaicin, at room temperature $\left(23^{\circ} \mathrm{C}\right)$, and also the set of temperature stimuli as shown in Table 1. Details of the rationale for the choice of the stimuli are given by Rolls et al. (2003b) and Verhagen et al. (2003c). The gustatory stimuli used included, $1.0 \mathrm{M}$ glucose $(\mathrm{G}), 0.1 \mathrm{M} \mathrm{NaCl}(\mathrm{N})$, $0.01 \mathrm{M} \mathrm{HCl}(\mathrm{H}), 0.001 \mathrm{M}$ quinine- $\mathrm{HCl}(\mathrm{Q})$, and $0.1 \mathrm{M}$ monosodium glutamate $(\mathrm{M})$. The concentrations of most of the tastants were chosen because of their comparability with our previous studies, and because they are in a sensitive part of the dose-response curve (Rolls et al. 1989; Scott et al. 1986b, 1991). Distilled water at $23^{\circ} \mathrm{C}$ was one member of the temperature series (T23) and with its viscosity of $1 \mathrm{cP}$ was also one member (V1) of the viscosity series. For an additional comparison, the neuronal responses were tested to $20 \%$ blackcurrant juice (BJ, Ribena) because with its complex taste and olfactory components and high palatability, it is an effective stimulus when searching for and analyzing the responses of cortical neurons (Rolls et al. 1990).

A viscosity series was made with carboxymethylcellulose (CMC, Sigma, high viscosity, MW: 700,000, dialysed, Code C5013), a virtually odor- and tasteless thickening agent used widely in the food industry (Rolls et al. 2003). Viscosity (or apparent viscosity given that $\mathrm{CMC}$ is non-Newtonian and shows some shear-thinning) was assessed using a calibrated Brookfield rotary viscometer (type LVT, Brookfield Engineering Laboratories, Middleboro, MA) at $60 \mathrm{rpm}$ (shear rate: $\sim 12 \mathrm{~s}^{-1}$, spindles $1-4$ ) at $23^{\circ} \mathrm{C}$. Concentrations (in g CMC added to $500 \mathrm{ml}$ water) yielding $1,10,100,1,000$, and $10,000 \mathrm{cP}$ (V1, V10, V100, V1000, and V10000; reliability: $\pm 10 \%$ ) solutions were: 0.0 , 0.1, 2.0, 5.5, and $12.0 \mathrm{~g} \mathrm{CMC}$, respectively (Theunissen and Kroeze 1995). The solutions were mixed until they were optically clear. Viscosity was assessed at room temperature after air bubbles had disappeared. (Note that $1 \mathrm{cP}=1 \mathrm{mPa} \mathrm{s}$ )

The gritty stimulus consisted of hard (Mohs scale 5) hollow microspheres (Fillite grade PG, with $87 \%$ having a diameter with the range 100-300 $\mu \mathrm{m}$, Trelleborg Fillite, Runcorn, UK) made up in methylcellulose to have a measured viscosity of $1,000 \mathrm{cP}$ (100 g of Fillite PG was added to $4.7 \mathrm{~g} \mathrm{CMC}$ in $500 \mathrm{ml}$ of water).

To test for and analyze the effects of oral fat on neuronal activity, a set of oils and fat-related stimuli was included. The triglyceridebased oils consisted of vegetable, safflower, and coconut oils. These were used to examine whether fat is represented by the responses of insular cortex neurons. Single cream (SC, $18 \%$ fat, viscosity: $12 \mathrm{cP}$, Coop brand, pasteurized) was used as an exemplar of a natural high fat content food of the type for which we wished to examine the neural representation and sensing mechanisms. All the neurons with fatrelated responses described in this and our earlier study (Rolls et al. 1999) responded well to single cream. Vegetable oil (VO, viscosity: $55 \mathrm{cP}$ at $23^{\circ} \mathrm{C}$ ), coconut oil ( $\mathrm{CO}$, viscosity: $40 \mathrm{cP}$ at $\left.23^{\circ} \mathrm{C}\right)$, and safflower oil ( $\mathrm{SaO}$, viscosity: $50 \mathrm{cP}$ at $23^{\circ} \mathrm{C}$, Aldrich), were used as natural high-fat stimuli. As Gilbertson and colleagues (Gilbertson 1998) had reported differential effects in isolated taste cells to linoleic and lauric acid in vitro, suggesting that the gustatory modality might be involved in orally sensing fat, we included (Verhagen et al. 2003c) in the stimulus set free linoleic $(\mathrm{LiA}, 100 \mu \mathrm{M})$ and lauric acid (LaA, $100 \mu \mathrm{M}$, sodium salt; Sigma) as well as oils rich in conjugated linoleic acid $(68-83 \%$ in the safflower oil) and lauric acid (coconut oil, CO, 45-50\%, 40 cP, Sigma) (Weiss 1983; Wills et al. 1998).

To investigate whether the neurons responsive to fatty-acid based oils were in some way responding to the somatosensory sensations elicited by the fat, stimuli with a similar mouth feel but nonfat 
TABLE 1. Stimuli

\begin{tabular}{|c|c|c|c|c|c|c|}
\hline Stimulus & Abbreviation & Concentration & MW & Temperature, ${ }^{\circ} \mathrm{C}$ & Viscosity, cP & Chemical Group \\
\hline Glucose & G & $1 \mathrm{M}$ & 180 & 23 & 1 & $\begin{array}{l}\text { Monosaccharide } \\
\text { aldohexose }\end{array}$ \\
\hline Black Currant J & BJ & $20 \%$ & & 23 & 1 & Mixture \\
\hline Monosodium & M & $0.1 \mathrm{M}$ & 187 & 23 & 1 & Amino acid salt \\
\hline \multicolumn{7}{|l|}{ Glutamate } \\
\hline $\mathrm{NaCl}$ & $\mathrm{N}$ & $0.1 \mathrm{M}$ & 58 & 23 & 1 & Inorganic salt \\
\hline $\mathrm{HCl}$ & $\mathrm{H}$ & $0.01 \mathrm{M}$ & 36 & 23 & 1 & Inorganic acid \\
\hline Quinine $\mathrm{HCl}$ & Q & $0.001 \mathrm{M}$ & 387 & 23 & 1 & Alkaloid \\
\hline Water & $\mathrm{T} 10$ & & & 10 & 1 & \\
\hline Water & $\mathrm{T} 23 / \mathrm{VI}$ & & & 23 & 1 & \\
\hline Water & T37 & & & 37 & 1 & \\
\hline Water & T42 & & & 42 & 1 & \\
\hline $\mathrm{CMC}$ & V10 & $0.2 \mathrm{~g}+11 \mathrm{VI}$ & 700,000 & 23 & 10 & Polysaccharide \\
\hline $\mathrm{CMC}$ & V100 & $4.0 \mathrm{~g}+11 \mathrm{VI}$ & 700,000 & 23 & 100 & Polysaccharide \\
\hline $\mathrm{CMC}$ & V1000 & $11.0 \mathrm{~g}+11 \mathrm{VI}$ & 700,000 & 23 & 1000 & Polysaccharide \\
\hline $\mathrm{CMC}$ & V10000 & $24.0 \mathrm{~g}+11 \mathrm{VI}$ & 700,000 & 23 & 10000 & Polysaccharide \\
\hline Gritty & $\mathrm{Gr}$ & $\begin{array}{l}100 \mathrm{~g} \text { Fillite }+ \\
9.4 \mathrm{~g} \mathrm{CMC}+ \\
11 \mathrm{VI}\end{array}$ & 700,000 & 23 & 1000 & $\begin{array}{l}\mathrm{SiO}_{2}+ \\
\quad \text { polysaccharide }\end{array}$ \\
\hline Mineral oil & MO & $100 \%$ & & 23 & 25 & $\begin{array}{l}\text { Hydrocarbon } \\
\text { mixture }\end{array}$ \\
\hline Silicone oil & $\mathrm{SiO} 10$ & $100 \%$ & & 23 & 10 & $\begin{array}{l}\text { Silicon-oxygen } \\
\text { polymer }\end{array}$ \\
\hline Silicone oil & $\mathrm{SiO} 100$ & $100 \%$ & & 23 & 100 & $\begin{array}{l}\text { Silicon-oxygen } \\
\text { polymer }\end{array}$ \\
\hline Silicone oil & $\mathrm{SiO} 1000$ & $100 \%$ & & 23 & 1000 & $\begin{array}{l}\text { Silicon-oxygen } \\
\text { polymer }\end{array}$ \\
\hline Vegetable oil & $\mathrm{VO}$ & $100 \%$ & & 23 & 55 & Fat \\
\hline Coconut oil & $\mathrm{CO}$ & $100 \%$ & & 23 & 40 & Fat \\
\hline Safflower oil & $\mathrm{SaO}$ & $100 \%$ & & 23 & 50 & Fat \\
\hline Single cream & $\mathrm{SC}$ & $100 \%$ & & 23 & 12 & Emulsion \\
\hline Lauric acid & $\mathrm{LaA}$ & $100 \mu \mathrm{M}$ & & 23 & 1 & $\mathrm{Ffa}$ \\
\hline Linoleic acid & LiA & $100 \mu \mathrm{M}$ & & 23 & 1 & Ffa \\
\hline Capsaicin & Cap & $10 \mu \mathrm{M}$ & & 23 & 1 & Vanillyl amide \\
\hline
\end{tabular}

chemical composition were used. These stimuli included paraffin/ mineral oil (pure hydrocarbon, viscosity $25 \mathrm{cP}$ at $23^{\circ} \mathrm{C}$, Sigma) and silicone oil $\left[\mathrm{Si}\left(\mathrm{CH}_{3}\right)_{2} \mathrm{O}\right)_{\mathrm{n}}, \mathrm{SiO}, 10,100$, and 1,000 cP (Brookfield viscometer calibration fluid)].

The temperature series was provided by water at $10^{\circ} \mathrm{C}$ (chosen as the cold stimulus - commercial cold drinks are served at $\left.6^{\circ} \mathrm{C}\right), 42^{\circ} \mathrm{C}$ (warm/hot but not noxious), $37^{\circ} \mathrm{C}$ (body temperature), and $23^{\circ} \mathrm{C}$ (room temperature). These temperature stimuli were produced by keeping the 10-ml applicator pipettes (described under stimulus delivery) in a $100-\mathrm{ml}$ bottle containing the same water as that inside the applicator pipette with the bottle itself maintained in a separate water bath controlled at 10,37 , and $42^{\circ} \mathrm{C}$ (T10, T37, and T42). As the temperature stimulus was delivered directly from the applicator to the mouth, there was no effect of the heat capacity of the applicator on the temperature of the water delivered to the mouth.

The capsaicin was made up as a $10 \mu \mathrm{M}$ solution (containing $0.3 \%$ ethanol). This is $\sim 15$ times the human recognition threshold of 0.66 $\mu \mathrm{M}$ (Szolcsanyi 1990).

The stimuli were kept in the dark at $-20^{\circ} \mathrm{C}$ for $\leq 1$ mo. After thawing they were used for $\leq 5$ days, stored overnight at $4^{\circ} \mathrm{C}$ in the dark. All fatty oils were kept in the dark under $\mathrm{N}_{2}$ at $4^{\circ} \mathrm{C}$ to avoid oxidation.

\section{Stimulus delivery}

The general method for stimulus delivery and accurate stimulus onset marking (Rolls et al. 1990) was modified by introducing repeater pipettes (Verhagen et al. 2003c). We used repeater pipettes (Eppendorf AG, Hamburg, Germany, type: Multipette Plus), and pipette tips (Combitips Plus, $10 \mathrm{ml}$ ), which were modified to allow the contact time of the fluid to provide a trigger pulse by using a screw to connect the lumen of the pipette to a stainless-steel cylinder round the pipette which in turn contacted an electrically conducting foam pad connected to a Schmitt trigger and against which the pipette rested. When fluid was expelled from the pipette and touched the tongue, the impedance to ground changed, and the pulse was triggered. We placed $10 \mathrm{~mm}$-long cones cut from $200 \mu \mathrm{l}$ Gilson pipette tips onto the tip of the repeater pipette tip, creating a fluid-free lumen, to prevent the system from being triggered when the tip touched the monkey's lips. For reliable triggering, a concentration of $5 \mathrm{mM} \mathrm{NaCl}$ was used to make the solution sufficiently conductive for the impedance system to trigger. [This concentration is well below the salivary $\mathrm{NaCl}+\mathrm{KCl}$ concentration of $\sim 25-30 \mathrm{mM}$ (Bartoshuk 1974; Guinard et al. 1998; Morino and Langford 1978; Nagler and Nagler 2001).] All watersoluble stimuli were thus made up to contain $5 \mathrm{mM} \mathrm{NaCl}$. Oil stimuli were triggered manually by touching the antistatic foam at the time of expelling the fluid from the tip. The tips were wiped clean before each stimulus presentation. For chronic recording in monkeys, a manual method for stimulus delivery is used because it allows for repeated stimulation of a large receptive surface despite different mouth and tongue positions adopted by the monkeys (Scott et al. 1986a,b). The stimulus application volume was $200 \pm 10 \mu \mathrm{l}$ because this is sufficient to produce large gustatory neuronal responses that are consistent from trial to trial and yet that do not result in large volumes of fluid being ingested that might, by producing satiety, influence the neuronal responses (Rolls et al. 1989, 1990).

The monkey's mouth was rinsed with $200 \mu \mathrm{l}$ T23/V1 (water) during the inter-trial interval (which lasted $\geq 30 \mathrm{~s}$ or until neuronal activity returned to baseline levels) between taste stimuli. The com- 
plete stimulus array was delivered in random sequence. Due to the tenacious nature of the oral coating resulting from the delivery of cream or of oil, and also for gritty and capsaicin, four $200-\mu$ l rinses with T23/V1 were given while allowing the subjects to swallow after each rinse. For V1000 and V10000, we used two such rinses. All the stimuli shown in Table 1 were delivered in permuted sequences with the computer specifying the next stimulus to be used by the experimenter. The spontaneous firing rate of the neuron was measured from trials in which no stimulus delivery occurred.

\section{Data analysis}

After cluster cutting of the spikes with Datawave software, the numbers of spikes of the single neuron in 80 time bins each $100 \mathrm{~ms}$ long starting at the onset of the stimulus were obtained using SPSS. Statistical analysis was performed on the numbers of spikes in the first 1-s period after stimulus onset, which was sufficiently long to include firing to even viscous liquids and sufficiently short so that lowviscosity taste stimuli were still activating the neurons as shown in Fig. 2 of Rolls et al. (2003b). An ANOVA was performed (with SPSS) to determine whether the neuron had significantly different responses to the set of stimuli. If the main ANOVA was significant, four further ANOVAs were performed to test for differences in neuronal responses among the set of taste stimuli (G, N, H, Q, M, and T23/V1) and among the members of the viscosity series V1-V10000, the set of fat stimuli (MO, $\mathrm{SiO} 10,100$, and $1000, \mathrm{VO}, \mathrm{CO}, \mathrm{SaO}$ ), and the set of temperature stimuli (T10-T42). Systat 10 was used for the generation of Pearson product-moment correlation coefficients calculated between the stimuli using the responses of all the neurons analyzed and graphical presentation of stimulus similarity using multi dimensional scaling (loss function: Kruskal; regression: mono) and cluster analysis (linkage: average, distance: Pearson).

A taste cell was defined by a significant effect in the ANOVA performed across the stimulus subset $(\mathrm{V} 1, \mathrm{G}, \mathrm{N}, \mathrm{M}, \mathrm{H}, \mathrm{Q})$ on the number of spikes during the first second after stimulus onset. Similarly, the viscosity cell criterion was based on a significant effect in the ANOVA between the set of stimuli V1-V10000. Fat cells were defined by a significantly larger average firing rate to the oils (viscosity: 25-100 cP) than to the average rates to V10 and V100 and by, in addition, a significant larger average firing rate to the oils than the spontaneous firing rate. The criterion for being sensitive to temperature was based on a significant effect in the ANOVA between the set of stimuli T10-T42. The critical alpha level was set at $P<0.05$. Further, the tests for capsaicin, lauric acid, and linoleic acid sensitivity were a two-tailed $t$-test comparing the responses of the neuron to these substances and to water. The test for gritty texture sensitivity was a 2 -tailed $t$-test comparing the responses of the neuron to the gritty texture stimulus (which has a viscosity of $1000 \mathrm{cP}$ ) and to the $1,000-\mathrm{cP}$ stimulus from the viscosity series made with CMC.

The breadth of tuning metric of Smith and Travers (1979) was calculated as follows. The proportion of a neuron's total response that is devoted to each of the four basic stimuli can be used to calculate its coefficient of entropy $(H)$. The measure of entropy is derived from information theory and is calculated as

$$
H=-k \sum_{i} p_{i} \log p_{i}
$$

where $H=$ breadth of responsiveness, $k=$ scaling constant (set so that $H=1.0$ when the neuron responds equally well to all stimuli in the set of size $n), p_{i}=$ the response to stimulus $i$ is expressed as a proportion of the total response to all the $n$ stimuli in the set. The coefficient ranges from 0.0 , representing total specificity to one of the stimuli, to 1.0 , which indicates an equal response to all of the stimuli. The sparseness of the representation $a$ can be measured (Rolls and Deco 2002; Rolls and Tovee 1995; Rolls and Treves 1998) by extending the binary notion of the proportion of neurons that are firing as

$$
a=\left(\sum_{i=1, N} r_{i} / N\right)^{2} / \sum_{i=1, N}\left(r_{i} / N\right)
$$

where $r_{i}$ is the firing rate of the $i$ th neuron in the set of $N$ neurons. The sparseness is within the range $0-1$ and assumes the value 0.5 for a fully distributed representation with binary encoding and $1 / \mathrm{N}$ for a local or grandmother cell representation with binary encoding. These measures of the fineness of the tuning of neurons are important in understanding the neuronal encoding of information (Rolls and Deco 2002; Rolls and Treves 1998).

\section{Screening cells}

While searching for neurons, we continuously applied samples from our stimulus set: G, N, Q, BJ, SC, VO, SO, V100, V1/T23, T10, and T42. We tested for olfactory responses using the odors vanilla, eugenol, naphthalene, or amyl acetate held close to the nostril on a perfumer strip (with a blank perfumer strip as a control) as this is an effective way of locating neurons with olfactory responses in, for example, the orbitofrontal cortex (Critchley and Rolls 1996; Rolls and Baylis 1994; Rolls et al. 1996). We also tested for visual responsiveness (to the sight of food, a saline associated square plaque, the approach of a taste stimulus toward the mouth, objects, faces, head movement, and lip-smacking) and auditory responsiveness (a $500-\mathrm{Hz}$ tone, coo-calls, grunts, and vocalization) as stimuli of these types do activate some amygdala neurons (Sanghera et al. 1979). When neurons were insensitive to these stimuli, we classified them as nonresponsive. Only cells responding consistently to at least one stimulus of the array were recorded, all stimuli being applied four to six times in permuted sequences.

\section{RES ULTS}

The data described in this paper were obtained in two monkeys. Of 1,122 screened neurons in the insular taste cortex, 62 neurons $(5.5 \%)$ responded in relation to oral viscosity, gritty texture, fat, taste, temperature, capsaicin, lauric acid, and/or linoleic acid; $1.5 \%$ of the sample of 1,122 neurons had responses related to mouth movements. [These neurons responded phasically whenever the monkey moved the mouth, could be made to fire when a control syringe containing no liquid touched the mouth and produced mouth movements, and have been described also by Scott and Plata-Salaman (1999). These neurons could have been somatosensory or motor, and were not part of the sample of 62 neurons with oral sensory responses considered below. The actual proportion of movement-related neurons could be higher than the $1.5 \%$ we found in that the aim of this investigation was to examine neurons with taste and/or somatosensory responses and then to examine whether these neurons had movement-related activity but not to search for movement-related neurons per se]. The remainder of the neurons were unresponsive to the oral stimuli used except for six neurons that increased their firing rates above the spontaneous level nonspecifically to all the stimuli applied. (In contrast to the 62 neurons described in detail here, they had no significant differential activity in a 1-way ANOVA that tested for differences among all the oral stimuli.)

\section{Insular taste cortex neurons with responses related to the viscosity of oral stimuli}

Figure 1 shows a neuron (bq112c2) with differential responses to viscosity within the $1-10,000 \mathrm{cP}$ CMC viscosity series [ANOVA within the viscosity stimuli; $F(4,16)=3.62$, 


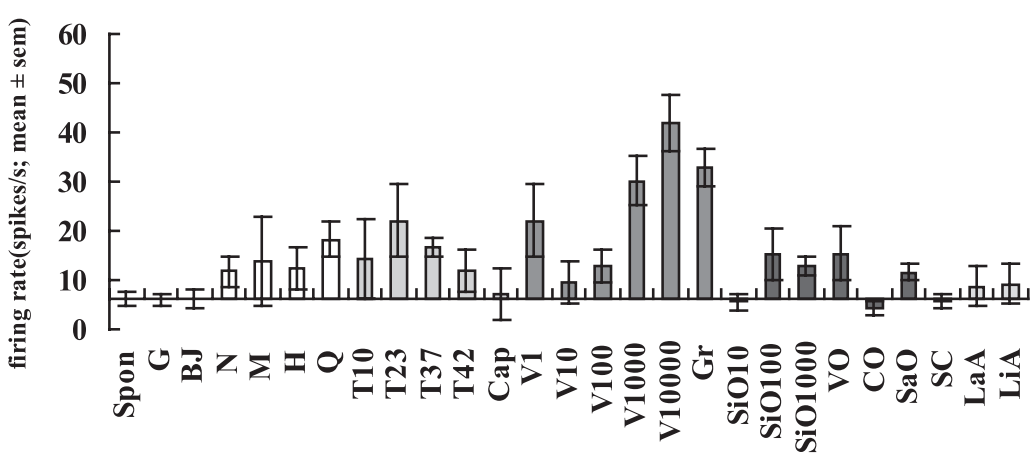

B

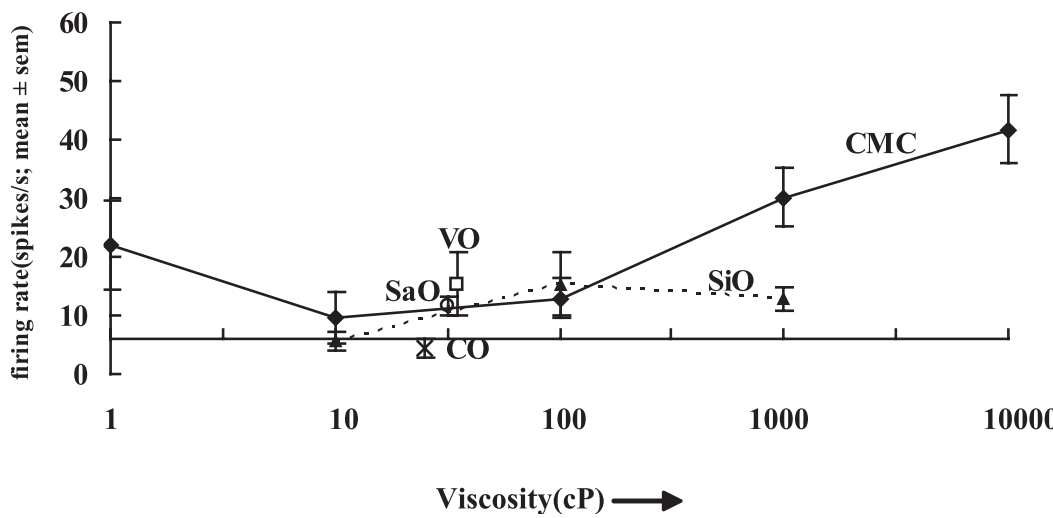

FIG. 1. A: responses of an insular taste cortex neuron (bq112c2) with differential responses primarily to viscosity as produced by the carboxymethylcellulose. The mean $\pm \mathrm{SE}$ firing rate responses to each stimulus calculated in a 1-s period over 4-6 trials are shown here and elsewhere unless otherwise indicated. The spontaneous (Spon) firing rate is shown by the horizontal line. The taste stimuli were $1 \mathrm{M}$ glucose $(\mathrm{G}), 0.1 \mathrm{M}$ $\mathrm{NaCl}(\mathrm{N}), 0.1 \mathrm{M}$ MSG (M), 0.01 M HCl (H), and $0.001 \mathrm{M}$ quinine- $\mathrm{HCl}(\mathrm{Q})$; the temperature stimuli were $\mathrm{T} 10, \mathrm{~T} 23, \mathrm{~T} 37$, and T42 where the number indicates the temperature in degrees celsius; the viscosity stimuli were V1, V10, V100, V1000, and $\mathrm{V} 10000$ where the numeral indicates the viscosity in centiPoise at $23^{\circ} \mathrm{C}$; fat texture stimuli were $\mathrm{SiO} 10, \mathrm{SiO} 100, \mathrm{SiO} 1000$ (silicone oil with the viscosity indicated), vegetable oil (VO), coconut oil (CO), and safflower oil ( $\mathrm{SaO})$. BJ. fruit juice; Cap, $10 \mu \mathrm{M}$ capsaicin; LaA, $0.1 \mathrm{mM}$ lauric acid; LiA, $0.1 \mathrm{mM}$ linoleic acid; Gr, the gritty stimulus (see Table 1) $B$ : the firing rate $( \pm 1 \mathrm{SE})$ to different viscosities of carboxymethylcellulose (CMC) shown as a graph, with the responses to the oils shown at their viscosity. The horizontal line shows the spontaneous activity.
$P=0.028]$. Figure $1 B$ shows the neuronal responses plotted as a function of the CMC viscosity value. The responses to the oils were generally as would be predicted from the CMC viscosity except that the response to $1,000 \mathrm{cP}$ CMC was larger than to $1,000 \mathrm{cP}$ silicone oil. (This smaller response to oils is a property found in some but not all of the texture-sensitive neurons as described in the following text.) This viscositysensitive neuron had no significantly different responses within the taste series nor within the temperature series. Further, it had no significant responses to the fatty acids $\mathrm{LiA}$ and $\mathrm{LaA}$ or to cream (SC). The neuron is thus an example of a unimodal viscosity-sensitive neuron in the anterior insular taste cortex. (The recordings sites of the different neurons will be shown later.)

The way in which each of the 33 viscosity-sensitive neurons responded to the different members of the viscosity series is shown in Fig. 2 in which the abscissa is the viscosity of the stimulus on a log scale for the five viscosity stimuli in the range $1-10,000 \mathrm{cP}$. For the purpose of ordering the neurons in Fig. 2, the neurons were grouped into five sets based on cluster analysis using the neuronal responses to V1-V10000. The first set of neurons in the diagram (1-15) tended to have decreasing firing rates as a function of viscosity. The second set of neurons in the diagram (16-23) tended to have increasing firing rates as a function of viscosity. The neurons in the next two groups (24-27, and 28-31) had firing rates that were tuned to oral viscosity.

Although some of the viscosity-sensitive neurons (i.e., the neurons with differential responses to the members of the $\mathrm{CMC}$ viscosity series as determined by ANOVA) responded to the silicone oil and the other oils in ways that would be predicted if they were responding to the viscosity of the oils, 11 of the 33 viscosity-sensitive neurons responded to the CMC viscosity series more (as shown by post hoc tests) than they responded to the equivalent viscosity when provided by an oil, and some (8) did not respond to the oil at all.

\section{Fat-responsive neurons}

Figure 3 shows a neuron ( $b q 88)$ that responded more to the set of oils than to the members of the viscosity series. Indeed, the response to the 10-, 100-, and 1,000-cP oils was greater than to the corresponding CMC viscosity [as shown by a main effect of oil vs. CMC in a 2-way ANOVA; $F(1,9)=27.48$, $P=0.001]$. [Within the CMC viscosity series, there were significant differences to the different viscosities; $F(4,8)=$ 9.46, $P=0.004)$, and this neuron was classified as being fat-responsive but also as being influenced by the $\mathrm{CMC}$ viscosity stimuli.] There was no significant response to any of the taste stimuli nor to any of the temperature stimuli. (The neuron did respond to $\mathrm{SC}$, a fat in water emulsion.) Interestingly, the neuron did not respond to the fatty acids $\mathrm{LaA}$ and $\mathrm{LiA}$, indicating that the responses to fat were based on its texture and not on any fatty acids that might possibly be present if fat is lipolysed at all in the mouth by any salivary lipase that might be present. Further evidence that the neuronal response was not based on fatty acids is that the neuron responded to the silicone oils (which contain no fat or fatty acids but have a similar texture to the fatty oils such as vegetable oil, $\mathrm{CO}$, and $\mathrm{SaO}$ ).

As shown in Table 2, five of the neurons (bq88, bol19, $b q 96$, bo154, and $b q 65 c 1)$ were fat sensitive in that their 

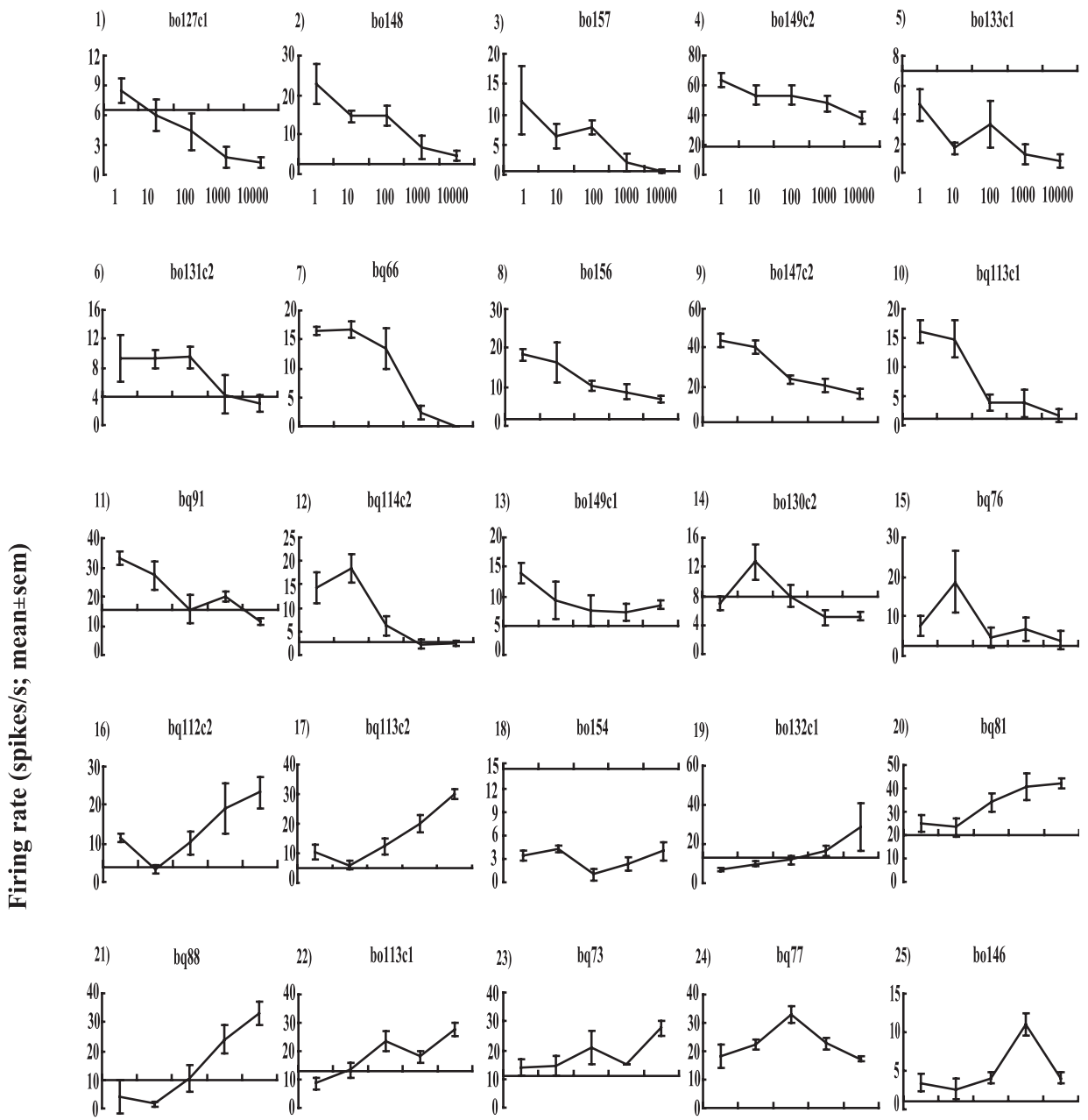

FIG. 2. The response functions of all the viscosity-sensitive neurons to $1,10,100$, 1,000 , and $10,000 \mathrm{cP}$ CMC. The mean $\pm \mathrm{SE}$ are shown. The spontaneous firing rate for each neuron is shown by the horizontal line.
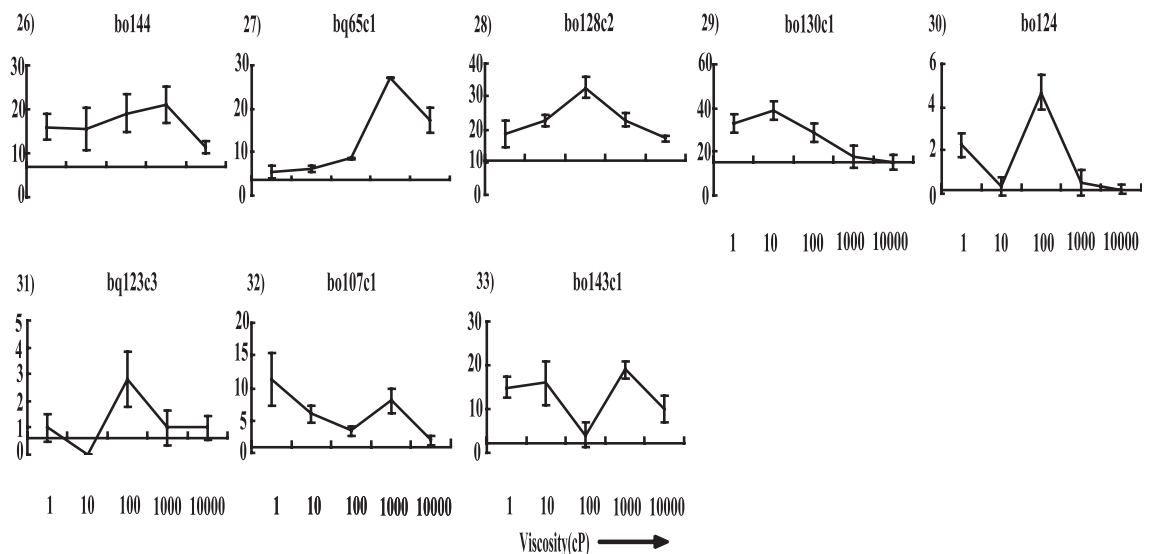

responses were large to the oils and occurred in a way that would not be predicted from any smaller response they might have to the CMC viscosity series (see example in Fig. 3 ). None of these five neurons responded to fatty acids. In addition, none of the 12 neurons with a response to one or both of the fatty acids were classified as fat sensitive. Ten of the 12 fatty-acid-sensitive cells responded to $\mathrm{HCl}$ and 2 did not, indicating that part of the responsiveness to the fatty acids could be related to the acid responsiveness of the neurons.

\section{Temperature-responsive neurons}

Figure 4 shows a neuron $($ bo $127 c l)$ with differential responses to different temperatures $\left[F(3,10)=37.43, P<10^{-4}\right]$. The neuron responded primarily to T10 from the temperature series with a small decrease of firing rate to T37 and T42 (this decrease being a response produced by many of the oral stimuli). The neuron did have a differential response to the set of taste stimuli [which included water, V1 $=\mathrm{T} 23$, to which the neuron had a small increase of firing rate; $F(5,17)=4.08, P=$ 
A

bq88

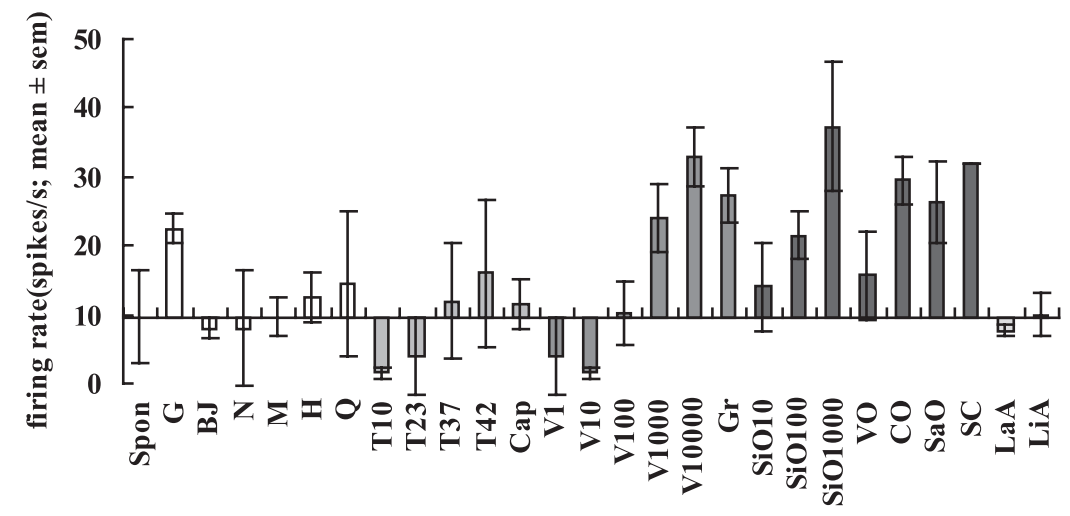

B

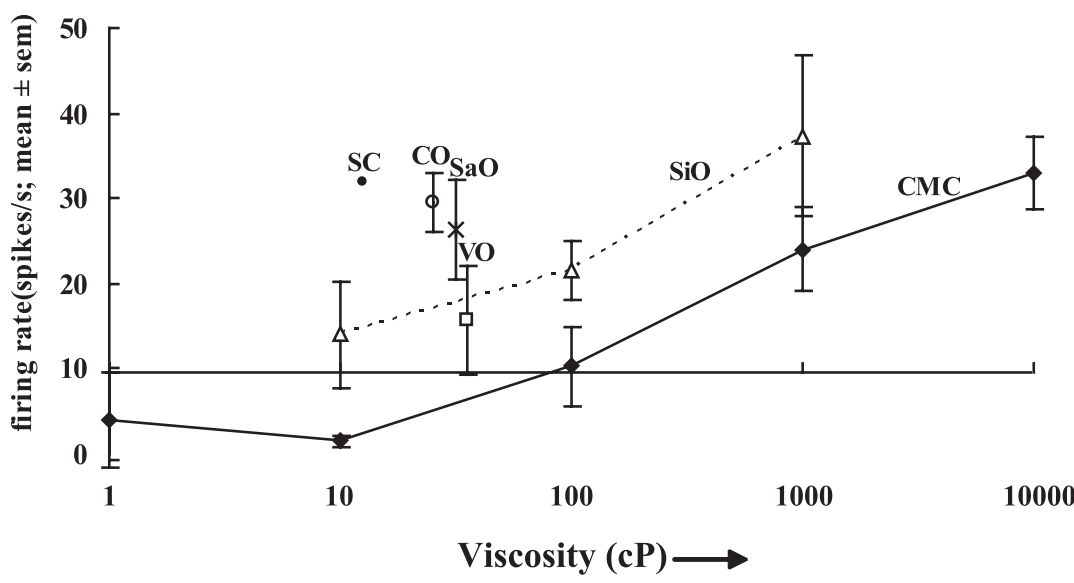

FIG. 3. A: responses of an insular taste cortex neuron (bq88) with larger responses to fatty oils and other oils than to carboxymethylcellulose. Conventions as in Fig. 1. $B$ : the firing rate $( \pm \mathrm{SE})$ to different viscosities of $\mathrm{CMC}$ shown as a graph with the responses to the oils shown at their viscosity. The horizontal line shows the spontaneous activity ( $\pm \mathrm{SE})$. Abbreviations as in Fig. 1.
0.013]. This neuron also had a differentially decreasing response as a function of viscosity $[F(4,15)=9.25, P=0.001]$.

The profiles of the responsiveness to the different temperature stimuli of the 22 oral thermosensitive neurons are shown in Fig. 5. The order of the neurons in Fig. 5 just for the purposes of illustration is based on four clusters identified by cluster analysis. In the first main cluster (1-13), the neuronal responses show a generally downward trend with increasing temperature (T10-42), and in the second main cluster (14-19), the neuronal responses show a generally upward trend with increasing temperature.

Of the 22 temperature-sensitive neurons, 2 responded only to temperature (see Table 2). Thus the temperature of what is in the mouth can be represented independently of taste, vis-

TABLE 2. Numbers of insular/frontal opercular neurons with different types of input

\begin{tabular}{lccc}
\hline \hline One Modality & Two Modalities & Three Modalities & Four Modalities \\
\hline G (15) & $\mathrm{G}+\mathrm{T}(6)$ & $\mathrm{G}+\mathrm{T}+\mathrm{V}(6)$ & $\mathrm{G}+\mathrm{T}+\mathrm{V}+\mathrm{F}(2)$ \\
$\mathrm{T}(2)$ & $\mathrm{G}+\mathrm{V}(6)$ & $\mathrm{G}+\mathrm{T}+\mathrm{F}(0)$ & \\
$\mathrm{V}(12)$ & $\mathrm{G}+\mathrm{F}(0)$ & $\mathrm{G}+\mathrm{V}+\mathrm{F}(0)$ & \\
$\mathrm{F}(2)$ & $\mathrm{T}+\mathrm{V}(6)$ & $\mathrm{T}+\mathrm{V}+\mathrm{F}(0)$ & \\
& $\mathrm{T}+\mathrm{F}(0)$ & & \\
& $\mathrm{V}+\mathrm{F}(1)$ & $(6)$ & \\
Total (31) & $(19)$ & & \\
\hline
\end{tabular}

Neuron numbers are in parentheses; G, taste; T, temperature; V, viscosity; $F$, fat. cosity, and fatty texture in the insular cortex. [Temperature can be represented independently in the sense that the finding of at least some different neurons for temperature with respect to taste, viscosity, and fat texture shows that separate, and therefore potentially independent, information channels are present. It is of course a further enterprise to show with information theoretic methods that these types of information are actually represented independently, but the methods are available for this (Franco et al. 2004; Rolls 2003a; Rolls and Deco 2002; Rolls et al. 2004).] In addition, 14 neurons responded to both taste and temperature, showing that the primate anterior insula represents combinations of these two modalities, potentially providing the basis for different behavioral responses to particular combinations of the taste and temperature of the food or fluid in the mouth. As shown in Table 2, six neurons in the primate insula responded to both oral temperature and oral viscosity, and in addition other neurons responded to temperature combined with several other types of oral sensory stimulus including taste and fat (see Table 2).

\section{Taste-responsive neurons}

Some neurons in the primate insular taste cortex had unimodal responses to taste. An example is shown in Fig. 6 [within taste; $F(5,24)=3.02, P=0.03$ ]. The neuron not only had no responses to viscosity, fat texture, and temperature but also, as shown in Fig. 6, had no responses to odor or to the 


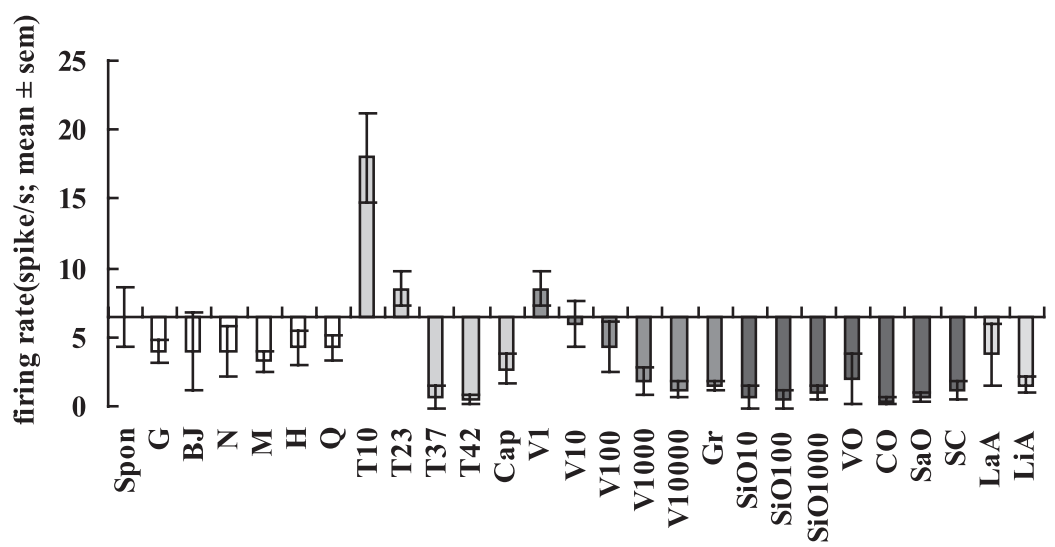

FIG. 4. The responses of an insular taste cortex neuron (bo127cl) responding primarily to temperature. Conventions as in Fig. 1. sight of food. Of the 35 taste-responsive neurons, 14 (40\%) responded to oral temperature and $14(40 \%)$ responded to oral texture (i.e., to viscosity or fat; see Table 2). Some of the taste neurons responded to both temperature and viscosity (see Table 2).

\section{Olfactory and visual response}

Of the 62 orally responsive neurons described in the preceding text, it was possible to test 25 for responses to olfactory or visual stimuli, and none had significant responses. An example of this type of result is shown in Fig. 7. The neuron had significantly different responses to different tastants $\left[F(5,20)=14.67, P<10^{-4}\right]$, no significant responses to oral viscosity, fat, or temperature, and no significant responses to any of the olfactory and visual stimuli tested (see Fig. 7). However, some (19) other neurons recorded in this insular region did have some responses to visual stimuli such as the sight of food approaching the mouth. As these neurons were not tested in a visual discrimination so that the latency of their neuronal response could be measured, it is possible that the activity of these neurons was related to anticipatory mouth movements made as the object approached the mouth. The activity of such neurons could have been related to somatosensory inputs occurring during small mouth movements, and indeed some other neurons (15) did respond to touch to the perioral region (e.g., the lips) or in two cases clearly in relation to mouth movements. No neurons in this cortical region responded to olfactory stimuli.

\section{Population analyses}

The representation of the similarity of the stimuli by the population of neurons was approached with multidimensional scaling analysis, based on the first $1 \mathrm{~s}$ of poststimulus activity and was performed on the responses of the same 62 neurons (Fig. 8). The segregations between modalities are clearly shown in the multidimensional space. The different modalities have been joined by lines to help clarify the representation in this multidimensional space. First, the viscosity series is very well separated in the space (primarily along the $x$ axis). The five taste stimuli are well separated from each other. The members of the temperature series are again clearly laid out in the space. The oils are located closely together and clearly separate from the viscosity series parametric representation. It is of considerable interest that the oil stimuli are not separated out in the space according to their viscosity as this provides further evidence that the viscosity of stimuli is encoded parametrically in the insular cortex and that fatty texture is coded as a fatty texture independently of its viscosity. Capsaicin and the fatty acids were not very well separated from water (T23/ V1).

The results of the cluster analysis on the same 62 neurons shown in the dendrogram in Fig. 9 is consistent with the MDS analysis. Although in general the correlations between different stimuli are relatively high (see Fig. 9) in the insular cortex, the stimuli that are very close to each other in the dendrogram indicate that the representation provided does represent real similarities between the stimuli with, for example, all the oils being close together in the dendrogram in a cluster that contains no CMC viscosity stimuli. Thus oral fat is very clearly distinguished from oral viscosity by the representation provided by the primate insula with the fats represented as a tight cluster. The viscosity stimuli are clearly separated from each other in the dendrogram, providing evidence that the primate insula provides a clear representation of viscosity. It is also evident in the dendrogram that T10 and T23 are well separated from each other and from T37/T42, showing that the population separates these three temperatures from each other well.

Of the 62 neurons in the sample, $31(50 \%)$ neurons were unimodal (15 unimodal taste, 2 unimodal temperature, 12 unimodal viscosity, and 2 unimodal fat neurons), 19 (31\%) neurons were bimodal, and $8(13 \%)$ neurons were multimodal with responses to taste, temperature, and viscosity (see Table 2 ). The findings provide clear evidence for convergence of taste and somatosensory (thermosensitive, texture sensitive, and/or fat sensitive) inputs onto some neurons in the insula (see Fig. 4) and also that each type of input is represented independently of the others (see Figs. 1, 3, and 6). Further, some neurons had responsiveness to $\mathrm{LaA}, \mathrm{LiA}$, and Cap when compared with their solvent water (T23/V1): 9 neurons to LaA, 4 neurons to $\mathrm{LiA}$ (with 1 of these neurons responding to both LaA and LiA), and 8 neurons to Cap. None of the fatty-acidsensitive neurons were classified as fat responsive. In addition, five neurons responded to the gritty texture stimulus (Gr; when compared with the equally viscous but not gritty V1000). 

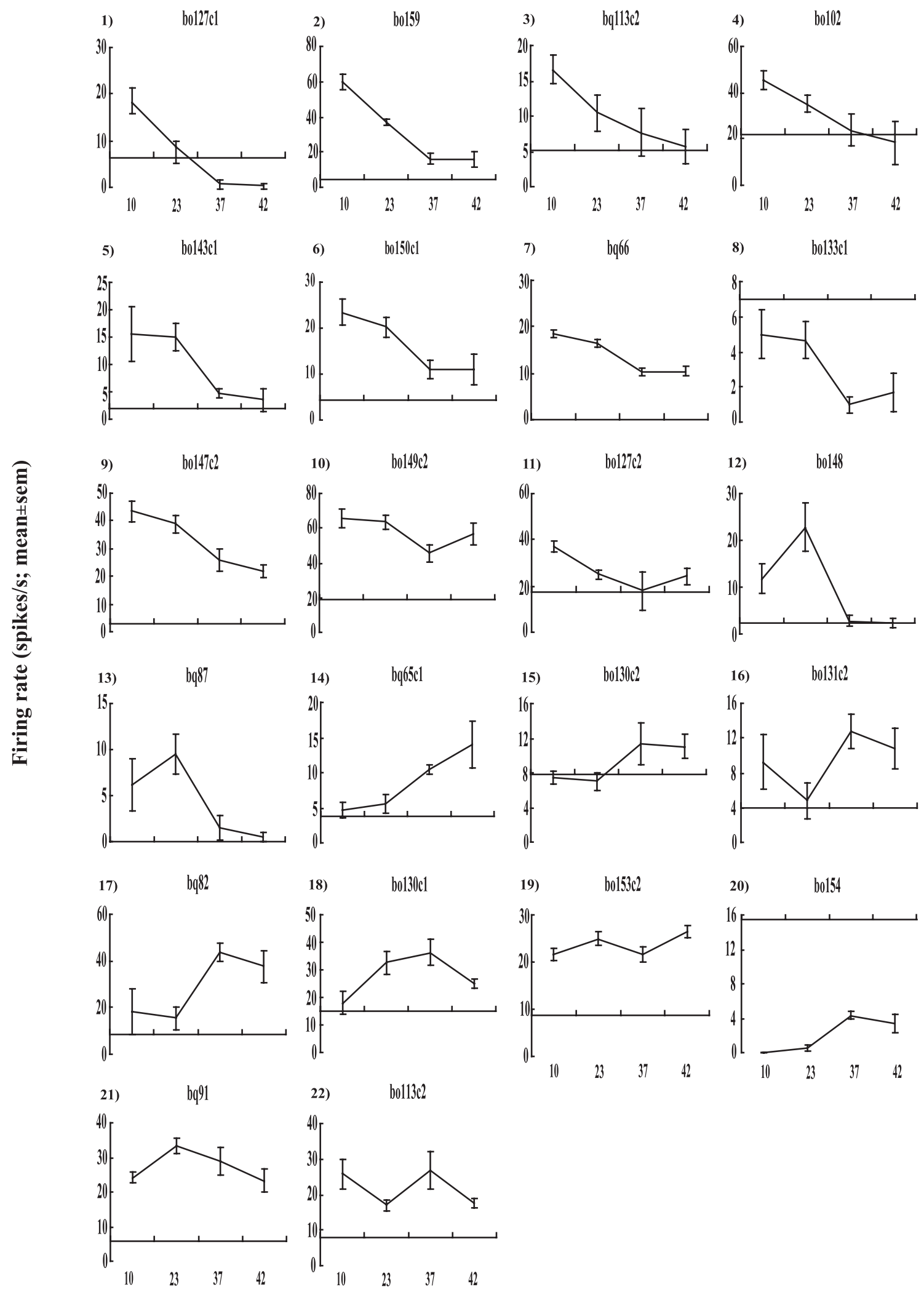

Temperature $\left(4^{\circ} \mathrm{C}\right) \longrightarrow$

FIG. 5. The response functions of all the oral thermosensitive neurons to different temperatures in degrees celcius (T10, T23, T37, and T42). The mean \pm SE are shown. The spontaneous firing rate for each neuron is shown by the horizontal line. 


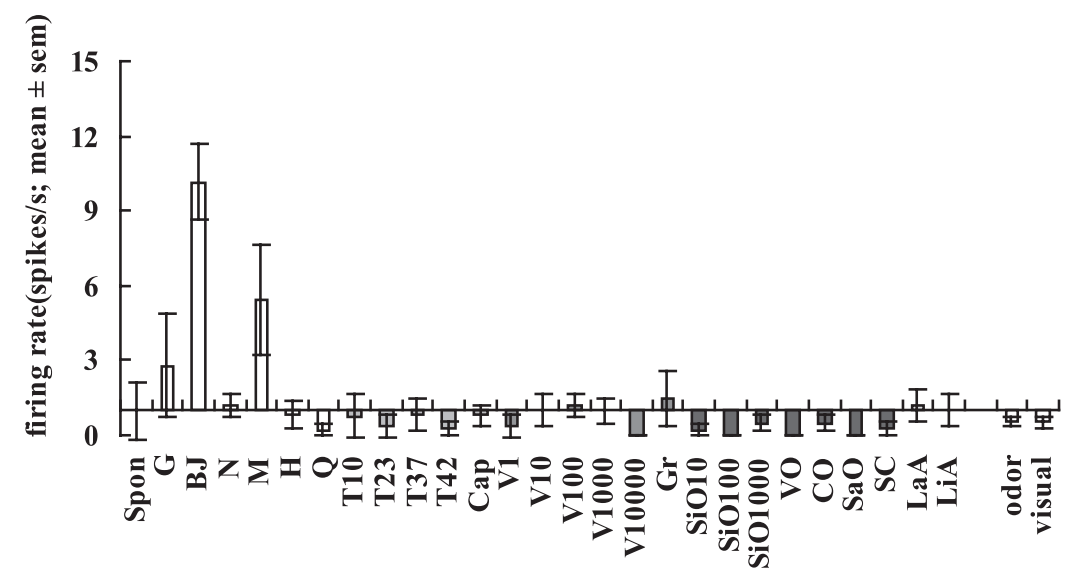

FIG. 6. The responses of an insular taste cortex neuron (bo135) with differential responses to taste but no responses to temperature, viscosity, fat, or any of the other stimuli. The neuron did not respond to odor or to the sight of food (visual). Conventions as in Fig. 1.
The breadth-of-tuning metric (Smith and Travers 1979) calculated across the taste stimuli $\mathrm{H}, \mathrm{Q}, \mathrm{N}$, and $\mathrm{G}$ was lower $(0.82 \pm 0.06$, mean $\pm \mathrm{SE}$; indicating finer tuning between the taste stimuli) for the neurons with only taste inputs (i.e., without somatosensory-thermosensitive, texture-sensitive, and/or fat-sensitive input) than for neurons with both taste and somatosensory inputs $(0.91 \pm 0.03)$ although this was not significant. The corresponding sparsenesses were $0.74 \pm 0.05$ and $0.85 \pm 0.04(P=0.08)$. In addition, the mean sparseness of the representation of 16 stimuli (G, BJ, N, M, H, Q, T23/V1, T10, T37, T42, V10, V100, V1000, SC, and VO) of the 62 insula neurons was $0.74 \pm 0.21$ (mean $\pm \mathrm{SD}$ ). This compares to the mean sparseness of 52 orbitofrontal cortex neurons to the same set of stimuli (Verhagen et al. 2003c) of $0.67 \pm 0.23$ $(P=0.12)$, which indicates the insular neurons were nonsignificantly tuned more broadly to the set of stimuli.

\section{Localization of recordings}

The reconstructed positions of the neurons analyzed in this study are shown in Fig. 10. All are within the region defined as primary taste cortex as shown by the cortical area receiving afferents from the thalamic taste nucleus VPMpc (Pritchard et al. 1986). It is notable though that the most posterior coronal section (3-4 $\mathrm{mm}$ posterior to sphenoid) shown in Fig. 10 contained some neurons with oral texture and/or temperature sensitivity but none with taste sensitivity, so this may be at the posterior boundary of the primary taste cortex.

\section{I S C U S S I O N}

The representation of viscosity described here encodes the degree of viscosity of what is in the mouth in that each neuron has graded firing to the different viscosities used (CMC in the range $1-10,000 \mathrm{cP}$ ) and in that different neurons have different response functions as shown in Fig. 2. Further evidence for this is provided by the multidimensional space shown in Fig. 8 in which the different viscosity stimuli are parametrically represented and well separated from each other in the stimulus space. The hard, round, microspheres we employed (100-300 $\mu \mathrm{m})$ evoke an oral gritty texture, and this was an effective stimulus when suspended in cellulose for one neuron (when compared with equally viscous cellulose).

Fat in the mouth was represented in two ways by the AI/FO taste cortex neurons described here. One way was by the $\mathrm{AI} / \mathrm{FO}$ taste cortex neurons that respond to fat and much less to the cellulose viscosity series (Fig. 3). These neurons encode fat by its texture (and not by any odor or free fatty acid cue) in that the same neurons respond to silicone oil, to mineral oil, and not to fatty acids (Gilbertson 1998; Verhagen et al. 2003c). The second way in which fat is distinguished from nonfat textures in the $\mathrm{AI} / \mathrm{FO}$ taste cortex is by the neurons that respond to viscosity and not to the oils (see example shown in Fig. 1). Indeed, it was of interest that most of the neurons differentially responsive to the cellulose viscosity series (11/33) tended to have smaller responses to the same viscosity when produced by fat, providing a further way in which the population of insular/

\section{bo139c2}

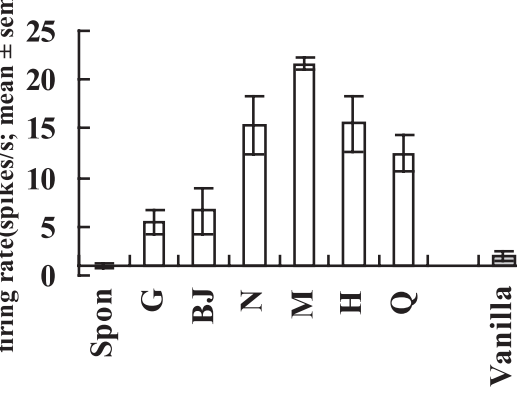

FIG. 7. A: responses of an insular taste cortex neuron (bo139c2) with taste responses to show the lack of response to a range of olfactory and visual stimuli. The visual stimuli were $\mathrm{BJ}$ syringe, the sight of a syringe containing fruit juice; $\mathrm{N}$ syringe, the sight of a syringe containing $0.1 \mathrm{~N} \mathrm{NaCl}$; forceps, control, showing the feeding forceps alone that were used to show and feed banana or peanut. Conventions as in Fig. 1. 


\section{$\mathrm{n}=62$}

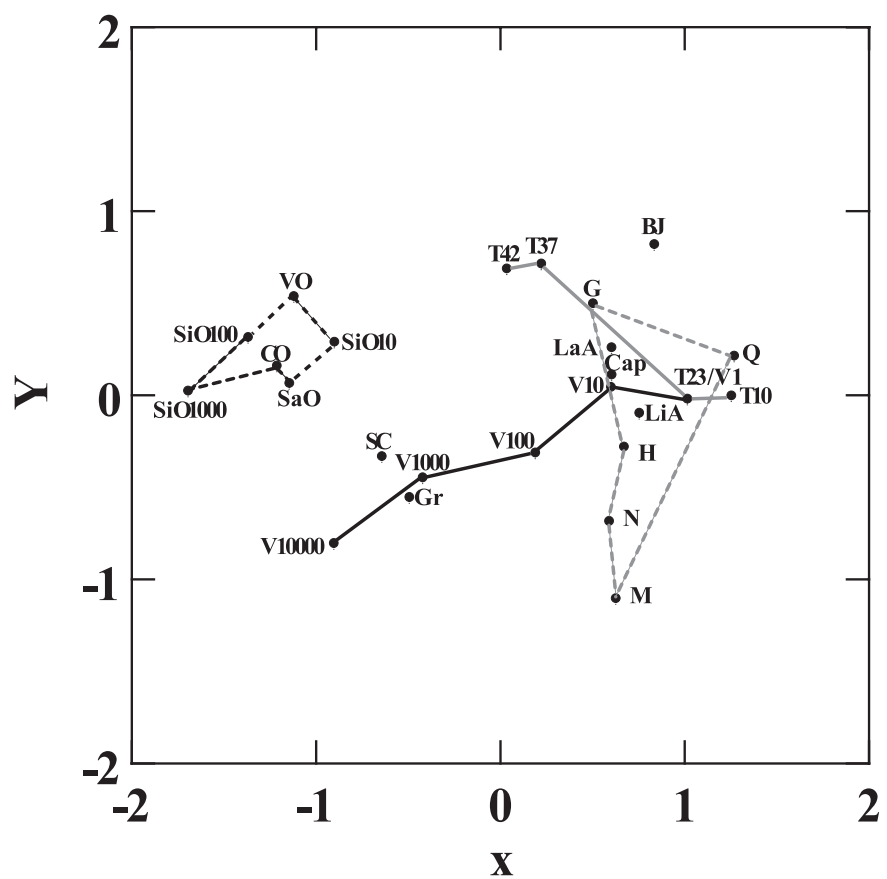

FIG. 8. A stimulus space (multidimensional scaling) of the stimulus similarity based on the across-neuron response profiles of the 62 orally responsive insular taste cortex neurons. The taste stimuli were $1 \mathrm{M}$ glucose $(\mathrm{G}), 0.1 \mathrm{M}$ $\mathrm{NaCl}(\mathrm{N}), 0.1 \mathrm{M}$ MSG (M), $0.01 \mathrm{M} \mathrm{HCl}(\mathrm{H})$, and $0.001 \mathrm{M}$ quinine- $\mathrm{HCl}(\mathrm{Q})$; the temperature stimuli were $\mathrm{T} 10, \mathrm{~T} 23, \mathrm{~T} 37$, and $\mathrm{T} 42$ where the number indicates the temperature in degrees celcius; the viscosity stimuli were V1, V10, V100, V1000, and V10000 where the numeral indicates the viscosity in centiPoise); fat texture stimuli were $\mathrm{SiO} 10, \mathrm{SiO} 100, \mathrm{SiO} 1000$ (silicone oil with the viscosity indicated), vegetable oil (VO), coconut oil (CO), and safflower oil $(\mathrm{SaO})$. BJ, fruit juice; Cap, $10 \mu \mathrm{M}$ capsaicin; $\mathrm{LaA}, 0.1 \mathrm{mM}$ lauric acid; $\mathrm{LiA}$, $0.1 \mathrm{mM}$ linoleic acid; Gr, the gritty stimulus. The solid line joins the members of the viscosity series. Different line styles join the members of the taste, temperature, and oil stimuli. The 2-dimensional solution accounted for $95 \%$ of the variance.

opercular neurons described here separates the representations of oral viscosity and fat. In addition, the few neurons that responded to fatty acids did not respond to the oil stimuli.

The representation of temperature provided by these $\mathrm{AI} / \mathrm{FO}$ taste cortex neurons was graded as shown by the responses of the neurons illustrated in Figs. 5 and 4 and by the multidimensional space shown in Fig. 8 in which the temperature stimuli are parametrically organized in the space. Four of the $62 \mathrm{AI} / \mathrm{FO}$ taste cortex orally responsive neurons tested in this study had responses to capsaicin that were different from water. The neurons did not respond to $42^{\circ} \mathrm{C}$ water, and this may be related to the fact that the sensation of capsaicin is mediated by the vanilloid receptor subtype 1 (VR1), which responds to temperatures $>43^{\circ} \mathrm{C}$ (Caterina et al. 1999).

Some of the AI/FO taste cortex neurons described here provide separate representations of viscosity, fat texture, temperature, taste, capsaicin, grittiness, and fatty acids, and other neurons combined inputs from different subsets of these properties of sensory stimuli. Some AI/FO taste cortex neurons responded to viscosity but not taste (31\%), some responded to taste but not viscosity (34\%), and other neurons responded to both viscosity and taste (23\%). Similarly, some AI/FO taste cortex neurons responded to temperature but not taste (13\%), some responded to taste but not temperature (34\%), and other neurons responded to both temperature and taste (23\%). The combination-responding neurons provide a basis for different behavioral responses to particular combinations of the sensory properties of stimuli such as food in the mouth. The fact that some $\mathrm{AI} / \mathrm{FO}$ taste cortex neurons respond to both taste and temperature shows that the temperature of what is in the mouth is not encoded only separately from the other sensory properties of the food but also in combination with other sensory properties of food. Thus this temperature representation may not only allow hot or cold substances to be rejected (or accepted) but also enables foods that have particular combinations of temperature, taste, and texture to be reacted to differently. In terms of proportions of neurons with unimodal versus bi- or multimodal inputs, the amygdala has similar proportions (52\%) (Kadohisa et al. 2004a) to the insular/opercular cortex $(50 \%)$, and the orbitofrontal cortex has relatively fewer unimodal neurons (30\%) (Rolls et al. 2003b).

The interesting finding that some primary taste cortex neurons respond to both taste and intra-oral somatosensory stimuli such as viscosity and temperature could reflect convergence in the insular cortex or the convergence could be present already at earlier stages of taste processing. It is known that some neurons in the taste thalamus (nucleus VPMpc) have thermal responsiveness in monkeys (Pritchard et al. 1989) and rats (Verhagen et al. 2003b). In the periphery, it is known that chorda tympani fibers in the monkey (Sato et al. 1975) and hamster (Ogawa et al. 1968) show significant correlations between the responses to $\mathrm{HCl}$ and those to cooling $\left(20^{\circ} \mathrm{C}\right)$ and between the responses to sucrose and warming (to $40^{\circ} \mathrm{C}$ ). Some lingual nerve fibers in monkeys were activated by

$$
\mathrm{n}=62
$$

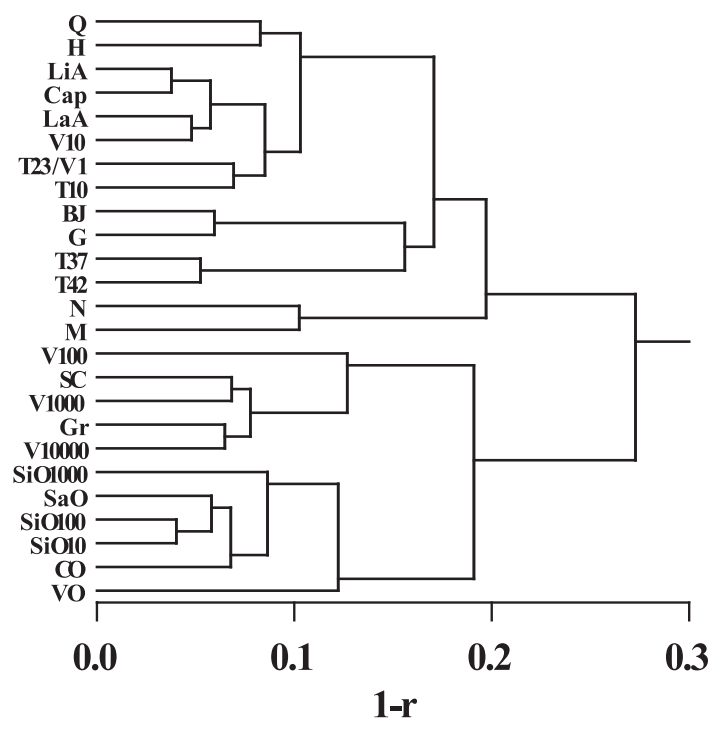

FIG. 9. A stimulus dendrogram based on the 62 orally responsive insular taste cortex neurons in the study. $(1-r)$ is the measure of dissimilarity of the clusters. ( $r$ is the correlation coefficient.) Abbreviations as for Fig. 8. 
0-1P

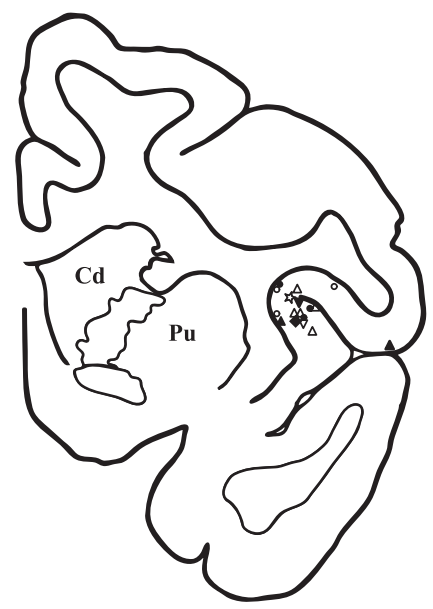

- gustatory

$\Delta$ viscosity

a temperature

* fat

- $\mathrm{g}+\mathbf{t}$

$\mathbf{g}+\mathbf{v}$
$\mathbf{t}+\mathbf{v}$

$\mathbf{t}+\mathbf{v}$
$\mathbf{v}+\mathbf{f}$

$\mathbf{g}+\mathbf{t}+\mathbf{v}$
$\mathbf{g}+\mathbf{t}+\mathbf{v}+\mathbf{f}$
1-2P

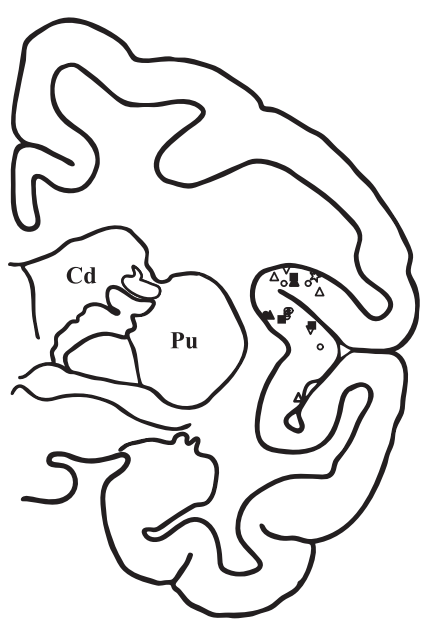

3-4P

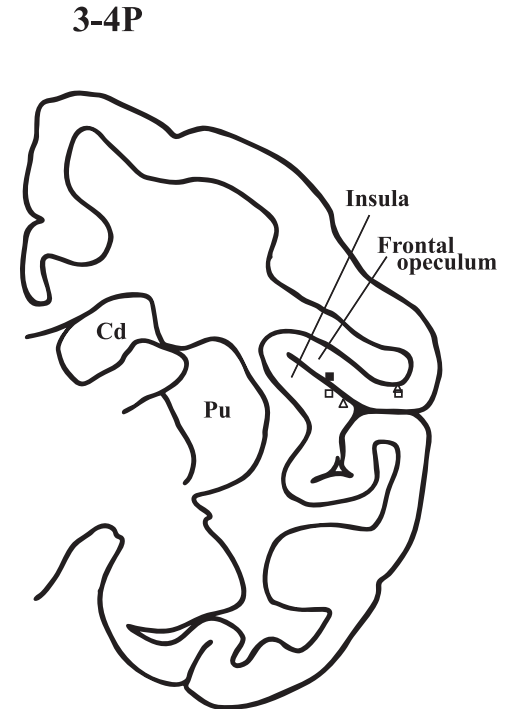

FIG. 10. The reconstructed positions in the brain of the neurons in this study. The coronal sections are at different distances in millimeters posterior $(\mathrm{P})$ to the sphenoid reference (which is at approximately the A-P level of the optic chiasm). The symbol with which the location of each neuron is indicated shows whether the neuron was tuned to $\mathrm{g}=$ taste, $\mathrm{v}=$ viscosity, $\mathrm{t}=$ temperature, $\mathrm{f}=$ fat or to combinations of these. $\mathrm{Cd}$, caudate nucleus; $\mathrm{Pu}$, putamen.

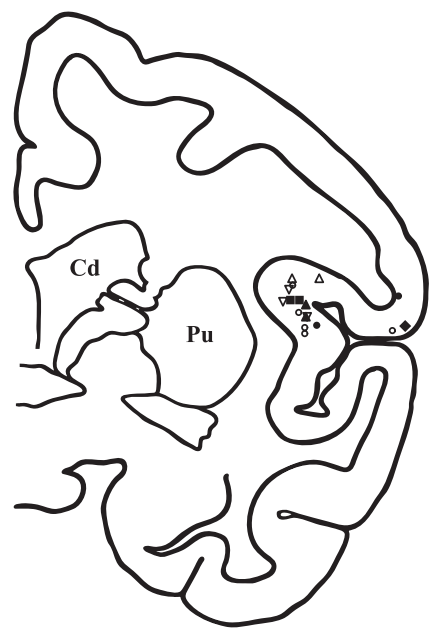

cooling to $15^{\circ} \mathrm{C}$ but not by taste (Danilova and Hellekant 2002). We know of no studies in the periphery of the effects of food-relevant oral stimuli such as viscosity and fat texture. It is also possible that oral somatosensory information reaches the AI/FO primary taste cortex via cortico-cortical connections, perhaps from area $3 \mathrm{~b}$, which contains oral somatosensory representations of for example touch of the tongue, teeth, and palate (Jain et al. 2001; Manger et al. 1996) and which might send afferents to the AI/FO cortex (Friedman et al. 1986; Mufson and Mesulam 1982).

Although the effects of intra-oral stimuli other than taste on primate primary taste cortex neurons have not been investigated previously as far as we know, there are reports that some neurons in the macaque insular cortex respond to tactile stimulation of the mouth region (Scott and Plata-Salaman 1999), although in the study of Schneider et al. (1993), none of these responded to taste. In the rat, there is some evidence that perioral mechanical and/or temperature (Kosar and Schwartz 1990a,b; Yamamoto et al. 1981, 1988) stimuli can activate some taste cortex neurons, but food-related oral stimuli such as texture were not investigated in those studies.
It was noticeable from the dendrogram (Fig. 8) that the inter-stimulus correlations across the population of 62 neurons were relatively high. Indeed, the mean correlation between pairs of the stimuli was $0.81 \pm 0.08$ (mean \pm SD) for the insular/opercular taste cortex. In comparison, the mean correlation across the same 20 stimuli for the orbitofrontal cortex was $0.71 \pm 0.12\left(t=10.5, \mathrm{df}=194, P<10^{-22}\right)$ and for amygdala neurons was $0.89 \pm 0.05(t=11.4 \mathrm{df}=194, P<$ $\left.10^{-26}\right)$. Thus a major characteristic of the processing beyond the primary taste cortex is that in the orbitofrontal cortex, the representation of oral stimuli is more distinct, that is, less correlated or more orthogonal. The same property is reflected in the sparsenesses of the representations, which for 16 stimuli (G, BJ, N, M, H, Q, T23/V1, T10, T37, T42, V10, V100, $\mathrm{V} 1000, \mathrm{SC}$, and VO) for the 62 insula neurons was $0.74 \pm$ 0.21 . This compares to the mean sparseness of 52 orbitofrontal cortex neurons to the same set of stimuli (Verhagen et al. $2003 \mathrm{c})$ of $0.67 \pm 0.23(P=0.12)$, which indicates the insular neurons were nonsignificantly tuned more broadly to the set of stimuli. For the same set of stimuli, the mean sparseness of 44 amygdala neurons (Kadohisa et al. 2004a) was $0.79 \pm 0.18$ 
$(P=0.16)$, which indicates the amygdalar neurons had a tendency to be more broadly tuned to the set of stimuli than the insular/ frontal opercular cortex neurons. Thus overall, this places the orbitofrontal cortex in a special functional role, for it sharpens the tuning of neurons to this broad range of oral stimuli, providing more separate representations of each oral stimulus. This more separate representation in the orbitofrontal cortex (OFC) than the insula or amygdala fits the OFC particularly well for functions such as sensory-specific satiety, which is computed in the OFC (Rolls et al. 1989) and not in the insular/frontal opercular primary taste cortex (Rolls et al. 1988; Yaxley et al. 1988). Sensory-specific satiety could be implemented by synaptic or neuronal adaptation (Deco and Rolls 2004) occurring over $10-15$ min of stimulation by a food, and the effect can only be relatively specific if the tuning of the individual neurons is relatively specific.

One factor contributing to the somewhat high value of 0.81 of the correlations between all stimuli for the $\mathrm{AI} / \mathrm{FO}$ cortex neurons was that the inter-stimulus correlations on which this mean correlation was based were calculated across 20 of the stimuli shown in Table 1. [These 20 stimuli included only one exemplar of the fats (VO), as the responses of the neurons to different oils were very similar]. We note that when calculating the correlations between the pairs of 20 stimuli, we do not subtract the spontaneous firing rate as the spontaneous firing rate would of course not be subtracted before the firing is transmitted to other neurons in the brain. When receiving neurons sum the postsynaptic potentials elicited through all the afferent synapses, the neuron has no way of distinguishing what is spontaneous from what is response-related neuronal input activity. Thus it is more realistic at the computational neuroscience level not to subtract the spontaneous rate (see further Rolls and Deco 2002; Rolls and Treves 1998), and this is why we present the correlation measures and the multidimensional scaling and cluster analyses without subtracting the spontaneous rate. However, we note for comparison with other studies (Rolls et al. 1988; Scott and Plata-Salaman 1999; Scott et al. 1986a, 1991; Yaxley et al. 1988, 1990) that the mean value of the correlations among the six taste stimuli $\mathrm{G}, \mathrm{N}, \mathrm{H}$, $\mathrm{Q}, \mathrm{T} 23 / \mathrm{V} 1$, and $\mathrm{BJ}$ for the taste responsive neurons with the spontaneous subtracted was $0.75 \pm 0.09$.

The separate (relatively uncorrelated) representations of different stimuli in the OFC may also be appropriate for a stage at which learning of associations between visual or olfactory stimuli and oral stimuli occurs (Critchley and Rolls 1996; Rolls et al. 1996; Thorpe et al. 1983) for then the learned association can reflect particular qualities of individual foods and other oral stimuli much more effectively. Indeed, one of the important findings of this investigation that is consistent with this hypothesis is that olfactory stimuli, and visual stimuli such as the sight of food, did not activate orally responsive neurons in the $\mathrm{AI} / \mathrm{FO}$ taste cortex, providing further evidence that this type of convergence (Baylis et al. 1994), which is implemented by associative learning (Critchley and Rolls 1996; Rolls et al. 1996; Thorpe et al. 1983), is an important function of the primate orbitofrontal cortex.

A number of functional neuroimaging studies have shown activation of an insular/frontal opercular cortical region by taste in humans (De Araujo et al. 2003b; O'Doherty et al. 2001; Small et al. 1999; Zald et al. 1998). In addition, a recent study has shown that the same insular/opercular region has BOLD
fMRI activation, which is correlated with the viscosity of carboxymethylcellulose, providing evidence that this region in humans, putatively the primary taste cortex, also receives an oral texture input (De Araujo and Rolls 2004). Of course, the details of the representation as described here, with both unimodal neurons, and bimodal neurons showing convergence, together with the details of the individual neuronal tuning to viscosity and temperature stimuli, and the separateness of the representation from gritty and capsaicin, could not be shown by fMRI studies. Another fMRI study does, though, also indicate that the results described at the neuronal level in primates are relevant to understanding the human insular cortical system. In particular, it was found that although the orbitofrontal cortex and the most anterior, agranular, insula in humans are activated by both taste and olfactory stimuli, there is a part of the human insular/frontal opercular cortex that is activated by only taste, and not by olfactory, stimuli (De Araujo et al. 2003a). We therefore believe that in both humans and macaques there is dorsally a part of the insular/opercular taste cortex that is not activated by olfactory stimuli and that at the transition between the more ventral part of the insular that is agranular and is topologically on the orbitofrontal surface, there is a region with taste and olfactory convergence. A diagram illustrating this is provided by De Araujo and Rolls (2004).

These results provide fundamental evidence about the information channels used to represent the taste, texture, and temperature of food in the first cortical area involved in taste in the primate brain. The current investigation thus greatly extends previous investigations in which taste representations in the primary taste cortex have been analyzed (Rolls et al. 1988; Scott and Plata-Salaman 1999; Scott et al. 1986a, 1991; Yaxley et al. 1988, 1990). The results are relevant to understanding the physiological and pathophysiological processes related to how the properties of oral stimuli are represented in the brain and thus to the control of food intake and food selection.

\section{A C K N OW LE D GMENTS}

We thank Drs. L. Engelen (Wageningen Centre for Food Sciences, The Netherlands) and F. Zoet (Wageningen University, The Netherlands) for measuring the size distribution of the gritty stimuli.

\section{G R A N T S}

This research was supported by Medical Research Council Grant PG9826105 to E. T. Rolls.

\section{REFERENCES}

Aggleton JP and Passingham RE. Stereotaxic surgery under X-ray guidance in the rhesus monkey, with special reference to the amygdala. Exp Brain Res 44: 271-276, 1981.

Bartoshuk LM. $\mathrm{NaCl}$ thresholds in man: thresholds for water taste or $\mathrm{NaCl}$ taste? J Comp Physiol Psychol 87: 310-325, 1974.

Baylis LL, Rolls ET, and Baylis GC. Afferent connections of the orbitofrontal cortex taste area of the primate. Neuroscience 64: 801-812, 1994.

Beckstead RM, Morse JR, and Norgren R. The nucleus of the solitary tract in the monkey: projections to the thalamus and brainstem nuclei. J Comp Neurol 190: 259-282, 1980.

Berthoud HR. Neural system controlling food intake and energy balance in the modern world. Curr Opin Clin Nutr Metabolic Care 6: 615-620, 2003.

Caterina MJ, Rosen TA, Tominaga M, Brake AJ, and Julius D. A capsaicin-receptor homologue with a high threshold for noxious heat. Nature 398: 436-441, 1999.

Critchley HD and Rolls ET. Olfactory neuronal responses in the primate orbitofrontal cortex: analysis in an olfactory discrimination task. J Neurophysiol 75: 1659-1672, 1996. 
Danilova V and Hellekant G. Oral sensation of ethanol in primate model III: responses in the lingual branch of the trigeminal nerve of Macaca mulatta. Alcohol 26: 3-16, 2002.

De Araujo IET, Kringelbach ML, and Rolls ET. Taste-olfactory convergence, and the representation of the pleasantness of flavour, in the human brain. Eur J Neurosci 18: 2374-2390, 2003a.

De Araujo IET, Kringelbach ML, Rolls ET, and Hobden P. The representation of umami taste in the human brain. J Neurophysiol 90: 313-319, 2003b.

De Araujo IET and Rolls ET. The representation in the human brain of food texture and oral fat. J Neurosci 24: 3086-3093, 2004.

Deco G and Rolls ET. Synaptic and spiking dynamics underlying reward reversal in orbitofrontal cortex. Cereb Cortex In press.

Feigenbaum JD and Rolls ET. Allocentric and egocentric spatial information processing in the hippocampal formation of the behaving primate. Psychobiology 19: 21-40, 1991.

Franco L, Rolls ET, Aggelopoulos NC, and Treves A. The use of decoding to analyze the contribution to the information of the correlations between the firing of simultaneously recorded neurons. Exp Brain Res 155: 370-384, 2004.

Friedman DP, Murray EA, O'Neill JB, and Mishkin M. Cortical connections of the somatosensory fields of the lateral sulcus of macaques: evidence for a corticolimbic pathway for touch. J Comp Neurol 252: 323-347, 1986.

Gilbertson TA. Gustatory mechanisms for the detection of fat. Curr Opin Neurobiol 8: 447-452, 1998.

Guinard J-X, Zoumas-Morse C, and Walchak C. Relation between parotid saliva flow and compostition and the perception of gustatory trigeminal stimuli in foods. Physiol Behav 63: 109-118, 1998.

Jain N, Qi H-X, Catania KC, and Kaas JH. Anatomic correlates of the face and oral cavity representations in the somatosensory cortical area $3 \mathrm{~b}$ of monkeys. J Comp Neurol 429: 455-468, 2001.

Kadohisa M, Rolls ET, and Verhagen JV. The primate amygdala: neuronal representations of the viscosity, fat texture, grittiness, and taste of foods in the mouth. European Journal of Neuroscience In press, 2004a.

Kadohisa M, Verhagen JV, and Rolls ET. Orbitofrontal cortex: neuronal representation of oral temperature and capsaicin in addition to taste and texture. Neuroscience 127: 207-221, 2004b.

Kosar E and Schwartz GJ. Effects of methanol on peripheral nerve and cortical unit responses to thermal stimulation of the oral cavity in the rat. Brain Res 513: 202-211, 1990b.

Manger PR, Woods TM, and Jones EG. Representation of face and intraoral structures in area $3 \mathrm{~b}$ of macaque monkey somatosensory cortex. J Comp Neurol 371: 513-521, 1996.

Morino T and Langford HG. Salivary sodium correlates with salt recognition threshold. Physiol Behav 21: 45-48, 1978.

Mufson EJ and Mesulam M-M. Insula of the old world monkey. II. Afferent cortical input and comments on the claustrum. J Comp Neurol 212: 23-37, 1982.

Nagler RM and Nagler A. The effect of pilocarpine on salivary constituents in patients with chronic graft-versus-host disease. Arch Oral Biol 46: 689-695, 2001.

Norgren R. Central neural mechanisms of taste. In: Handbook of Physiology. The Nervous System. Sensory Processes. Washington, DC: Am. Physiol. Soc., 1984, sect. 1, vol. III, p. 1087-1128, 1984.

Norgren R. Taste pathways to hypothalamus and amygdala. J Comp Neurol 166: $17-30,1976$.

Norgren R and Leonard CM. Ascending central gustatory pathways. J Comp Neurol 150: 217-238, 1973.

O'Doherty J, Rolls ET, Francis S, Bowtell R, and McGlone F. The representation of pleasant and aversive taste in the human brain. J Neurophysiol 85: 1315-1321, 2001.

Ogawa H, Sato M, and Yamashita S. Chorda tympani fibers of the rat and hamster to gustatory and thermal stimuli. J Neurophysiol 199: 223-240, 1968.

Pritchard TC, Hamilton RB, Morse JR, and Norgren R. Projections of thalamic gustatory and lingual areas in the monkey, Macaca fascicularis. J Comp Neurol 244: 213-228, 1986.

Pritchard TC, Hamilton RB, and Norgren R. Neural coding of gustatory information in the thalamus of Macaca mulatta. J Neurophysiol 61: 1-14, 1989.

Pritchard TC, Macaluso DA, and Eslinger PJ. Taste perception in patients with insular cortex lesions. Behav Neurosci 113: 663-671, 1999.
Rolls ET. The Brain and Emotion. Oxford, UK: Oxford Univ. Press, 1999.

Rolls ET, Aggelopoulos NC, Franco L, and Treves A. Information encoding in the inferior temporal cortex: contributions of the firing rates and correlations between the firing of neurons. Biol Cybern 90: 19-32, 2004.

Rolls ET and Baylis LL. Gustatory, olfactory, and visual convergence within the primate orbitofrontal cortex. J Neurosci 14: 5437-5452, 1994.

Rolls ET, Critchley HD, Browning AS, Hernadi A, and Lenard L. Responses to the sensory properties of fat of neurons in the primate orbitofrontal cortex. J Neurosci 19: 1532-1540, 1999.

Rolls ET, Critchley HD, Mason R, and Wakeman EA. Orbitofrontal cortex neurons: role in olfactory and visual association learning. J Neurophysiol 75: 1970-1981, 1996.

Rolls ET and Deco G. Computational Neuroscience of Vision. Oxford, UK: Oxford Univ. Press, 2002.

Rolls ET, Franco L, Aggelopoulos NC, and Reece S. An information theoretic approach to the contributions of the firing rates and correlations between the firing of neurons. J Neurophysiol 89: 2810-2822, $2003 \mathrm{a}$.

Rolls ET, Scott TR, Sienkiewicz ZJ, and Yaxley S. The responsiveness of neurones in the frontal opercular gustatory cortex of the macaque monkey is independent of hunger. J Physiol 397: 1-12, 1988.

Rolls ET, Sienkiewicz ZJ, and Yaxley S. Hunger modulates the responses to gustatory stimuli of single neurons in the caudolateral orbitofrontal cortex of the macaque monkey. Eur J Neurosci 1: 53-60, 1989.

Rolls ET and Tovee MJ. Sparseness of the neuronal representation of stimuli in the primate temporal visual cortex. J Neurophysiol 73: 713-726, 1995.

Rolls ET and Treves A. Neural Networks and Brain Function. Oxford, UK: Oxford Univ. Press, 1998.

Rolls ET, Verhagen JV, and Kadohisa M. Representations of the texture of food in the primate orbitofrontal cortex: neurons responding to viscosity, girttiness and capsaicin. J Neurophysiol 90: 3711-3724, 2003b.

Rolls ET, Yaxley S and Sienkiewicz ZJ. Gustatory responses of single neurons in the caudolateral orbitofrontal cortex of the macaque monkey. J Neurophysiol 64: 1055-1066, 1990.

Sanghera MK, Rolls ET, and Roper-Hall A. Visual responses of neurons in the dorsolateral amygdala of the alert monkey. Exp Neurol 63: 610-626, 1979

Sato M, Ogawa H, and Yamashita S. Response properties of macaque monkey chorda tympani fibers. J Gen Physiol 66: 781-821, 1975.

Schneider RJ, Friedman DP, and Mishkin M. A modality-specific somatosensory area within the insula of the rhesus monkey. Brain Res 621: 116-120, 1993.

Scott TR and Plata-Salaman CR. Taste in the monkey cortex. Physiol Behav 67: 489-511, 1999.

Scott TR, Plata-Salaman CR, Smith VL, and Giza BK. Gustatory neural coding in the monkey cortex: stimulus intensity. J Neurophysiol 65: 76-86, 1991.

Scott TR, Yaxley S, Sienkiewicz ZJ, and Rolls ET. Gustatory responses in the frontal opercular cortex of the alert cynomolgus monkey. J Neurophysiol 56: 876-890, 1986a.

Scott TR, Yaxley S, Sienkiewicz ZJ, and Rolls ET. Taste responses in the nucleus tractus solitarius of the behaving monkey. J Neurophysiol 55: 182-200, 1986b.

Small DM, Zald DH, Jones-Gtman M, Zatorre RJ, Pardo JV, Frey S, and Petrides M. Human cortical gustatory areas: a review of functional neuroimaging data. Neuroreport 10: 7-14, 1999.

Smith DV and Travers JB. A metric for the breadth of tuning of gustatory neurons. Chem Senses 4: 215-219, 1979.

Steinberger J and Daniels SR. Obesity, insulin resistance, diabetes, and cardiovascular risk in children. Circulation 107: 1448-1453, 2003.

Szolcsanyi J. Capsaicin, irritation, and densensitisation: neurophysiological basis and future perspective. Chem Senses 2: 141-168, 1990.

Theunissen MJM and Kroeze HA. The effect of sweetners on perceived viscosity. Chem Senses 20: 441-450, 1995.

Thorpe SJ, Rolls ET, and Maddison S. Neuronal activity in the orbitofrontal cortex of the behaving monkey. Exp Brain Res 49: 93-115, 1983.

Turner BH, Mishkin M, and Knapp M. Organization of the amygdalopetal modality-specific cortical association areas in the monkey. J Comp Neurol 191: 515-543, 1980.

Verhagen JV, Gabbott PL, and Rolls ET. A simple method for reconditioning epoxy-coated microelectrodes for extracellular single neuron recording. J Neurosci Methods 123: 215-217, 2003a. 
Verhagen JV, Giza BK, and Scott TR. Responses to taste stimulation in the ventroposteromedial nucleus of the thalamus in rats. J Neurophysiol 89: 265-275, 2003b.

Verhagen JV, Rolls ET, and Kadohisa M. Neurons in the primate orbitofrontal cortex respond to fat texture independently of viscosity. J Neurophysiol 90: 1514-1525, 2003c.

Weiss TJ. Food Oils and Their Uses. Chichester, UK: Horwood, 1983.

Wills WM, Lencki RW, and Marangoni AG. Lipid modification strategies in the production of nutritionally functional fats and oils. Crit Rev Food Sci Nutr 38: 639-674, 1998.

Yamamoto T, Matsuo R, Kiyomitsu Y, and Kitamura R. Sensory input from the oral region to the cerebral cortex in behaving rats: an analysis of unit responses in cortical somatosensory and taste area during ingestive behaviour. J Neurophysiol 60: 1303-1321, 1988.

Yamamoto T, Yuyama N, and Kawamura Y. Cortical neurons responding to tactile, thermal and taste stimulations of the rat's tongue. Brain Res 221: 202-206, 1981.

Yaxley S, Rolls ET, and Sienkiewicz ZJ. The responsiveness of neurons in the insular gustatory cortex of the macaque monkey is independent of hunger. Physiol Behav 42: 223-229, 1988.

Yaxley S, Rolls ET, and Sienkiewicz ZJ. Gustatory responses of single neurons in the insula of the macaque monkey. J Neurophysiol 63: 689-700, 1990.

Zald DH, Lee JT, Fluegel KW, and Pardo JV. Aversive gustatory stimulation activates limbic circuits in humans. Brain 121: 1143-1154, 1998. 